\title{
SCHEMATA: THE CONCEPT OF SCHEMA IN THE HISTORY OF LOGIC
}

\author{
JOHN CORCORAN
}

\begin{abstract}
Schemata have played important roles in logic since Aristotle's Prior Analytics. The syllogistic figures and moods can be taken to be argument schemata as can the rules of the Stoic propositional logic. Sentence schemata have been used in axiomatizations of logic only since the landmark 1927 von Neumann paper [31]. Modern philosophers know the role of schemata in explications of the semantic conception of truth through Tarski's 1933 Convention T [42]. Mathematical logicians recognize the role of schemata in first-order number theory where Peano's second-order Induction Axiom is approximated by Herbrand's Induction-Axiom Schema [23]. Similarly, in first-order set theory, Zermelo's second-order Separation Axiom is approximated by Fraenkel's first-order Separation Schema [17]. In some of several closely related senses, a schema is a complex system having multiple components one of which is a template-text or scheme-template, a syntactic string composed of one or more "blanks" and also possibly significant words and/or symbols. In accordance with a side condition the template-text of a schema is used as a "template" to specify a multitude, often infinite, of linguistic expressions such as phrases, sentences, or argument-texts, called instances of the schema. The side condition is a second component. The collection of instances may but need not be regarded as a third component. The instances are almost always considered to come from a previously identified language (whether formal or natural), which is often considered to be another component. This article reviews the often-conflicting uses of the expressions 'schema' and 'scheme' in the literature of logic. It discusses the different definitions presupposed by those uses. And it examines the ontological and epistemic presuppositions circumvented or mooted by the use of schemata, as well as the ontological and epistemic presuppositions engendered by their use. In short, this paper is an introduction to the history and philosophy of schemata.
\end{abstract}

\$1. Introduction. In some of several closely related senses, a schema (plural: schemata, or schemas), also known as a scheme (plural: schemes), is a complex system having several components one of which is a template-text or scheme-template, a syntactic string composed of one or more "blanks" and usually also words and/or symbols to which meanings are assigned. The template-text of a schema is used as a "template" to specify a multitude, often infinite, of linguistic expressions such as phrases, sentences, argumenttexts or proof-texts. The collection of specified expressions, called instances

Received July 30, 2005. 
of the schema, may but need not be regarded as another of its components. The instances ${ }^{1}$ are almost always considered to come from a previously identified language (whether formal or natural), which is often considered to be another integral component of the schema.

Each schema is accompanied, implicitly or explicitly, with a side condition specifying how the "blanks" (dummies, placeholders, variables or ellipses) are to be "filled" to obtain instances, and also, sometimes, how the other words or symbols are to be understood [42, p. 55]. This side condition may also be regarded as another component of the schema or schematic system, as it may appropriately be called. Characters and character strings commonly used as blanks include: a "space", a dash, a short line, three dots, three dashes, dot-dash-dot, and so on. But there are many other strings used as blanks: ordinal number expressions ('the first', 'the second', etc.), letters of various alphabets, numerals, circled characters, and boxed characters, to mention a few other possibilities. It is important to note that the side condition does not assign a denotation to the blanks nor does it assign them even the "meaning" normally attached to a variable. In short, blanks are mere place-holders; they do not "range over a domain".

More precisely, the template-text of a given schema is a string of characters each of which is in the alphabet of the underlying object language or is an auxiliary character. As suggested above, the auxiliary characters are called blanks, place-holders, dummies, or other similar term (cf. [49, p. 473]). Regrettably, they are also misleadingly referred to as (syntactic or metalanguage) variables.

There are two limiting cases of such strings over an extended alphabet: (1) no blanks, a string of characters of the object language, and (2) nothing but blanks. The strings falling under the first limiting case are simply object language expressions, not template-texts. ${ }^{2}$ The strings falling under the second limiting case are rather strange - they are simply strings of blanks, auxiliary characters. In this case it would be awkward to use a "space" for a

\footnotetext{
${ }^{1}$ The word 'instance' is ambiguous. With regard to schemata it is unambiguous: in order for an expression from the underlying language to be an instance of a given schema it is necessary and sufficient for that expression to be a member of the set specified by that schema. In other connections, however, the word 'instance' is used as a relation noun for any one of several other relations. For example, it is often used as a synonym for 'token' in Peirce's sense of a concrete inscription. But here an instance of a schema is not a concrete string-token but an abstract string-type, again in Peirce's sense. Peirce's type-token distinction dates from the first few years of the 1900s. Although he made the distinction earlier, his use of the words 'type' and 'token' to express it dates from the 1906 Monist article on pragmaticism [35], quoted in Ogden-Richards [33, Appendix D, Section 6, esp. pp. 280-281] and reprinted in volume IV of Collected Papers [36, Paragraph 537]. See below for more on type-token ambiguity.

${ }^{2}$ This point is just a convenient technical convention. Imagine using the sentence ' $0=1$ ' is true iff $0=1$ ' with the side condition that both occurrences of ' $0=1$ ' are to be filled by occurrences of one and the same English sentence.
} 
blank. Surprisingly perhaps, many of these turn up as template-texts in some interesting and familiar historically important applications to be mentioned below.

Schema T. Among the best-known schemas is Tarski's schema T, also known as the schema (T), whose template-text is the eight-word two-blank string: ' . . . is a true sentence if and only if ...'. The side condition requires that the second blank is to be filled in with a (declarative) sentence of English and the first blank is to be filled in by a name of that sentence [42, p. 155]. The following two strings are instances - the first uses a quotes-name of the sentence filling the second blank, the second uses a phonetic descriptive name:

'zero is one' is a true sentence if and only if zero is one zee-ee-ar-oh-space-igh-es-space-oh-en-ee is a true sentence if and only if zero is one

More revealing instances are obtained by using a sentence not known to be true and not known to be false: 'every perfect number is even' is a true sentence if and only if every perfect number is even. Another such instance is: 'some perfect number is odd' is a true sentence if and only if some perfect number is odd. ${ }^{3}$

Defining the notion. Usage varies and can be controversial, and there is much that is not clear or not known. Moreover, no generally recognized definition or characterization of a concept of schema is known to the author. Consequently, it would be premature, and ultimately unhelpful, to attempt a single, strict, formally and materially adequate definition of "schema" at this point, not to mention an even more demanding analytically adequate definition that would "capture some definite concept". Nevertheless, it may be heuristically useful to consider possible tentative, informal definitions that seem to be presupposed in the literature.

The literature seems to confirm that in many cases a schema, or schematic system, may be construed as a four-part system (or ordered quadruple) consisting of a scheme-template, a side condition, an underlying language, and a set of instances. Other construals may be found to be convenient for various purposes. For example, a schema may be identified with the three-part system (ordered triple) composed of the scheme-template, the side condition, and the underlying language. The latter construal may be found especially attractive to those who wish to emphasize that the set of instances is determined by the three-part system composed of the schemetemplate, the side condition and the underlying language. Some logicians

\footnotetext{
${ }^{3}$ The meticulous accuracy and discipline needed in this field is illustrated by the fact, often ignored, that Tarski's side condition implicitly requires that the initial capitalization and final period (full stop) not be considered part of the sentence. For example, the sentence is 'zero is even', not 'Zero is even', not 'zero is even.' and certainly not 'Zero is even.'. Some people never grasped this point (Quine [39, p. 12]).
} 
may sometimes seem to identify the schema with the template alone or with the set of instances alone, or with the two-part system composed of the scheme template and either the side condition or the underlying language. Of course, nothing precludes the side condition from including specification of the underlying language.

The wording used by Tarski [42, pp. 155-156, esp. p. 156] suggests that he identifies the schema or scheme (his word) with the schema-template. A decade later, using the word 'schema', he is more explicit that it is an expression, a string, that he calls a schema [45, p. 344]; he says that the "expression" here called a schema-template "is not a sentence, but only a schema of a sentence". Incidentally, this is the first occurrence of the word 'schema' in [45]. It is clear that 'schema' is not being introduced as a technical term but rather that it is being used as a familiar locution of standard English. This terminology was also adopted by other logicians (e.g., Linsky [28, p. 3]) and it continues to be used (e.g., Goldfarb [21, p. 37]). In contrast, the Church wording [6, p. 149] seems calculated to avoid such identification of the schema with the schema-template. Vaught, in his influential 1967 paper [49] "Axiomatizability by a Schema", defines the unitary expression 'is axiomatizable by a schema' without defining 'schema' at all, even though his remarks using the word make perfect sense read in the senses in focus in this paper. However, none of the logicians mentioned, in fact none that I know of, directly raise the issue of whether a schema is or is not to be regarded simply as a string of characters. It is worth explicitly noting that there can be no objective criticism of identifying a schema with a schema-template this is a matter of terminology and nothing more.

It is clear that one and the same scheme-template may be a component of any number of different schemata depending on the side condition or on the underlying language or on both. ${ }^{4}$ Some of the same authors who write as though the schema is to be identified with the scheme-template alone also use one and the same scheme-template in connection with different underlying languages and thus with different schemes. Furthermore, since different characters are used for blanks (see above) and since even one notational change produces a different syntactic string in the strict sense (Corcoran [12]), one and the same set of instances may be determined by different scheme-template/side-condition pairings even given a fixed language. It may be this fact that leads some authors to write as though the scheme is to be identified with the set of instances. For many purposes it is the set of specified instances that is of primary importance and the question of exactly

\footnotetext{
${ }^{4}$ In order for "two" strings to be identical (that is, not two) it is necessary and sufficient for "them" to be character-by-character identical: to have the same characters occurring in the same order. This commonplace point is not so easy to find in the literature, but Rosenbloom [40, p. 152] has a clear statement of it: "Two strings are said to be the same if they have the same length and the same signs in corresponding places".
} 
what is involved in specifying it is considered a mere technicality. Each such construal or identification corresponds to a different sense of the ambiguous word 'schema'.

Pre-logical usage. Speakers of English are well aware that the nouns 'schema', 'scheme' and their cognates are parts of normal English and that non-logical uses of these words predated their incorporation as technical terms into logic. Since stipulated technical usage is powerless to completely cancel metaphorical implications, overtones and connotations accompanying ordinary usage, it will be useful to review, however briefly, a contemporary lexicographic treatment of them. According to the authoritative 2000 Merriam-Webster's Collegiate Dictionary [30], the noun 'scheme' entered the language in 1610 almost 300 years before 'schema' which is dated "ca. 1890". Moreover the older word 'scheme' was borrowed from Latin where it meant "arrangement, figure". In contrast, the newer word 'schema' was borrowed from Greek where it was cognate with the verb "echein to have, to hold, to be in (such) a condition". The older noun 'scheme' has been used in connection with mathematical diagrams, graphic sketches and outlines, concise statements and tables, plans of action (especially those which are crafty or secret) and systematic organized frameworks or designs. The words 'design' and 'plan' are among the synonyms listed for 'scheme'. Use of the younger noun 'schema', again according to this dictionary, has been much more limited: aside from a technical use in cognitive psychology totally irrelevant to present concerns, its only use listed is for "a diagrammatic presentation; broadly: a structured framework or plan". The word 'outline', literally "a line that marks the outer limits of an object or figure", is the only synonym listed for 'schema'. References to other dictionaries will be made below as relevant.

The rest of the paper is organized as indicated in the list below.

2. Examples of schemas.

3. The ontological status of schemas.

4. Schemas in the history of logic.

5. Argument-texts and argument-text schemas. Bibliography.

§2. Examples of schemas. Excluded-Middle. The fourteen-word sentence 'either zero is even or it is not the case that zero is even' is an instance of the excluded-middle sentence schema for English, which involves the eightword-two-blank string 'either ... or it is not the case that ...' '. The side condition is that the two blanks are to be filled by occurrences of the same well-formed English declarative sentence, that the discontinuous expression 'either ... or ...' expresses classical non-exclusive "or" and that the six-word sentence-prefix 'it is not the case that' expresses classical negation. Notice that this scheme-template is not an English sentence and it would be strictly 
speaking incoherent to use it as a sentence in an attempted assertion. Using another method to indicate blanks would not affect this point.

For example, instead of using the three-dot row as the blank it is equally correct to use a letter such as pee or kyu as in the eight-word-two-letter string:

$$
\text { either } p \text { or it is not the case that } p
$$

with the side condition that the two blanks are to be filled by occurrences of the same well-formed English declarative sentence, that the discontinuous expression 'either ... or ...' expresses classical non-exclusive "or" and that the six-word sentence-prefix 'it is not the case that' expresses classical negation. The schema-template resembles in some ways the string that Tarski [43, p. 38], [44, p. 33] uses to express what he calls the law of identity:

\section{If $p$, then $p$.}

But Tarski has already introduced an abbreviational convention according to which initial universal quantifiers are omitted. Thus the string that Tarski is using to express his law of identity is strictly speaking not the above but rather:

\section{For every sentence $p$, if $p$, then $p$.}

It is clear that pee is not a blank but a "sentential variable" whose range is the class of whatever sentences denote or have as "values".

However, the proper part of the schema-template devoid of blanks is literally a discontinuous part of each of its instances and, in fact, what all instances have in common is having this discontinuous string-like entity as a part. It is only in the strictest and most rigorous contexts where a distinction is to be made between, for example, the discontinuous two-word expression 'if ... then ...' and the continuous two-word two-blank scheme-template indicated by the same string.

Naming. As another example, the seven-word sentence 'the expression 'zero' names the entity zero' is an instance of the naming schema for English, which involves the five-word-two-blank string 'the expression ... names the entity ...', where the second blank is to be filled by a proper name of an entity and the first blank is to be filled by a proper name of a proper name of the entity. ${ }^{5}$ Another instance of the naming schema is 'the expression zeeee-ar-oh names the entity 0', where 'zee-ee-ar-oh' is an orthographic proper name of the four-letter word 'zero', which is a proper name of the entity 0 , i.e., zero. The twelve-character string 'zee-ee-ar-oh' in this usage is similar to the Tarskian structural-descriptive name of the four-letter word 'zero' as in the 1933 truth-definition paper [42, pp. 157, 160, 172].

\footnotetext{
${ }^{5}$ Notice that the first blank may but need not be filled with a name of the expression filling the second blank. The previous example illustrates the "may" and the next example illustrates the "need not". These and related points were suggested by Ricardo Santos (per. comm.).
} 
Use and mention. As Tarski points out, phonetic or other similar descriptive devices permit a much sharper distinction between "use" and "mention" than the quotes-name method. In perhaps the most direct senses of the expressions 'uses' and 'mentions', in every case the quotes-name method uses an expression in order to mention it. As Frege said [19 n 261]: "As ... proper names of the sentences of the object-language I u hese very sentences, but enclosed in quotation marks". In a phono-orthographic method it is only in rather atypical cases that an expression is used in order to mention it. For example, zee-ee-ee names zee. The last letter of the alphabet is the first letter of its own name. Normally the expression mentioned is not used. In the instance 'the expression zee-ee-ar-oh names the entity zero' it is clear that 'zero' is used but not mentioned in the object position, whereas it is mentioned but not used in the subject position.

However, in order to make an analogous point about the instance 'the expression 'zero' names the entity zero' it is necessary to stipulate a less natural sense of 'uses' or to make the claim, called metaphysical and even theological by non-logicians, that although the subject of the sentence is the six-character string quote-zee-ee-ar-oh-quote, which the sentence uses, the sentence does not use the four-character string zee-ee-ar-oh (Tarski [47, pp. 104-105]). ${ }^{6}$

However, if we use the expression 'word' in one of the familiar rich senses in which a word is something that, for example, is inherently meaningful and that might have more than one spelling or more than one meaning, then there is a lot more to using a word than just using the string that spells it. When I use the English word spelled see-aitch-ay-tee, I am not thereby using the English words aitch-ay-tee, ay-tee, and ay. And I am not thereby using the French word spelled see-aitch-ay-tee, the German word aitch-ay-tee, and the Spanish word see-aitch-ay. Similarly, in a straight-forward sense of 'uses', not every sentence using the eight-letter word 'recenter' uses the four-letter word 'cent'. But, it would invite criticism to say that one can use the string word 'recenter' without using the string 'cent'.

Tarski's Original Template-text. The example given in the introductory section above, Tarski's schema $\mathrm{T}$, warrants further discussion. In the form mentioned above, the template-text for Tarski's schema $\mathrm{T}$ is the eight-word two-blank string: ' $\ldots$ is a true sentence if and only if ... '. In its original 1933 formulation Tarski used ecks for the first blank and pee for the second: $x$ is a true sentence if and only if $p$

The side condition includes the requirement that the second blank is to be filled in with a sentence of English and the first blank is to be filled in by a name of that sentence (Tarski [42, p. 155]). It is clear that ecks and pee are

\footnotetext{
${ }^{6}$ Frango Nabrasa (per. comm.) said: "This act of using would be like eating an entire hard-boiled egg without eating the yolk, something only slightly less miraculous than the Monotheistic Trinity".
} 
place-holders, that ecks is not a "sentential-name variable" and that pee is not a "sentential variable." Tarski's 1944 template-text is a slightly different string but it raises no new theoretical issues. However, his 1969 template-text uses the letter pee as the only blank.

"p" is a true sentence if and only if $p$

What is remarkable here is that the three character expression quote-peequote is not being used as a quotes name of the letter pee. However, Tarski's formulation of the side condition contains the following wording [47, p. 105]:

where " $p$ " is to be replaced on both sides

which does use quote-pee-quote as a quotes name of the letter pee. Tarski's 1944 template-text contains the following discontinuous constituent:

"..." is a true sentence if and only if ...

whose first gap follows the first quote and whose second gap follows the second occurrence of igh-eff. Later in the same article Tarski uses quoteess-quote in two senses: for the quotes name of the letter ess, but not for a gap enclosed in quotes, rather for the quotes name of a long sentence that is abbreviated by ess [47, p. 108].

Sentence. Although Tarski does not say so, it is clear that the word 'sentence' is taken in the sense of the clausal form of sentence which does not end with a period (or full stop) and does not begin with an uppercase unless its initial word is capitalized. For example, 'zero is one' is the clausal form of the sentence whose assertoric form is 'Zero is one.' ending with a period or "full stop". By an assertoric form of sentence is meant a string of characters that is or could normally be used to make an assertion. By a clausal form sentence is meant a string of characters that is or could normally be used as a proper part of an assertoric form. ${ }^{7}$

Further, in the 1933 article Tarski [42] is not using the word 'sentence' in the modern sense of an "uninterpreted sentence" or "closed formula" (Boolos [4, p. 103]), rather he intends, in his words [42, p. 178], a "meaningful sentence" that says something in virtue of which it has a truth-value. Tarski is using the word 'sentence' in exactly the same sense that traditional postscholastic logicians attached to the word 'proposition', according to Church [6, p. 26, and fn. 68]. ${ }^{8}$ In Tarski 1933, a sentence is a string of characters that is true or is false as it stands; in Boolos et al 2002 a sentence per se is

\footnotetext{
${ }^{7}$ Although Frege [19, p. 261] introduces no new terminology nor does he note the initial capitalization and final period, he does note other differences between assertoric and clausal forms in German, viz. word order. In word-for-word translation it would be 'Jan is a man' and 'Jan is mortal', but 'If Jan a man is, is Jan mortal'.

${ }^{8}$ This is not the sense of 'proposition' that Church prefers. In his preferred sense, a proposition per se has no wording but can be expressed in many different wordings in different languages. Davis [13, p. xxii] wrote: "The fundamental property of a statement is that it is asserts a proposition that must be true or false". Thus, he seems to follow Church's preferred use of 'proposition' while using 'statement' as Tarski uses 'sentence'.
} 
not something that is either true or false, rather it is either true under a given interpretation or false under that interpretation.

Moreover, 'if and only if' is to be taken in the truth-functional sense and not as involving any modality or necessity. ${ }^{9}$ In order for an instance of Tarski's schema $\mathrm{T}$ to be modal it is necessary and sufficient for the sentence on the right of the "principal" occurrence of 'if and only if' to be modal. The important point here is that an instance of Tarski's schema $\mathrm{T}$ does not say that anything is necessarily the case. For example the instance mentioned above says that 'zero is one' is a true sentence if and only if zero is one. It does not say that it is necessarily the case that 'zero is one' is a true sentence if and only if zero is one.

Circularity and Quotes-names. Tarski's schema T, which he calls a scheme not a schema, is of special philosophical significance since, according to Tarski and many others, each instance of the schema is a "partial definition of truth", or rather of "true sentence". Accordingly, the naming schema may be regarded as giving partial definitions of the concept of naming, or rather of the relational concept "names".

Phonetic naming of alphabetic and other characters, 'ay' for the first letter, 'bee' for the second, and so on, was a commonplace in ancient written Greek and Latin but is curiously rare in written English except for its prominence in the 1933 truth-definition paper and in the literature deriving from it. Its advantages continue to be exploited in the best of contemporary logical writing (cf. e.g., Boolos [4, pp. 102, 107]). It is common, but strictly speaking incorrect, to talk of the name of a sentence or other string; each string has many names. Tarski [42, pp. 156, 157], [47, pp. 104, 105] features two systems of string naming, both already used above: the quotation-mark name, as 'zero' and the structural-descriptive name, as zee-ee-ar-oh. But, other systems are widely used, e.g., the italic name, as the proper name zero and the common noun thing (Church [6, p. 8]). The structural-descriptive or phonetic-name method, which Tarski characterizes as "a letter-by-letter description" [47, p. 105], is preferred by Tarski because it avoids the "appearance of a vicious circle" when an instance of Tarski's schema is regarded as a definition as mentioned above. Compare the following three instances of the naming schema.

the expression 'zero' names the entity zero

the expression zee-ee-ar-oh names the entity zero

the expression zero names the entity zero

The first has two occurrences of the string zee-ee-ar-oh, i.e., of the string 'zero', one in the subject and one in the direct object, an apparent circularity or triviality. The second has only one, in the direct object; thus avoiding

\footnotetext{
${ }^{9} \mathrm{I}$ do not recall seeing this point in print by Tarski or by anyone else. However, Tarski uniformly avoids modal assertions. He even goes so far as to construe ' $\mathrm{Q}$ is a consequence of P' as the non-modality "every model of P is a model of Q".
} 
any appearance of circularity or triviality (Tarski [47, pp. 104-105]). The reader may have either opinion on the question of whether the third has an occurrence in its subject.

$\S 3$. Ontological status of schemas. It is important to be clear about the mixed ontological status of schemas. In the first place, the associated string of words, symbols, etc., and blanks, which was called the template-text of the schema for emphasis, is a syntactic object, a string of characters, which involves the same ontological presuppositions as numerals, words, formulas, and the like. As seen above the template-text for the English naming schema, 'the expression ... names the entity ...' is a forty-character expression involving twenty-seven letter-occurrences, six occurrences of the space, and seven occurrences of the period. In the second place each of the instances of a schema is another syntactical object. The forty character string 'the expression 'ten' names the entity 10' is an instance of the naming schema for English. But the side conditions are intensional entities comparable to propositions in the traditional abstract sense (Church [6, pp. 26, 27], CohenNagel [7, pp. xxii, xxiii]) or abstract truth conditions, as suggested by Wm. Demopoulos (per. comm.).

Type-token-occurrence. Further discussion of the ontology of schemas requires Peirce's type-token distinction. The following is from Peirce's 1906 Monist article [35, pp. 504-505] quoted ${ }^{10}$ in Ogden-Richards [33, pp. 280281].

A common mode of estimating the amount of matter in a ... printed book is to count the number of words. There will ordinarily be about twenty 'thes' on a page, and, of course, they count as twenty words. In another sense of the word 'word,' however, there is but one word 'the' in the English language; and it is impossible that this word should lie visibly on a page, or be heard in any voice .... Such a ... Form, I propose to term a Type. A Single ... Object ... such as this or that word on a single line of a single page of a single copy of a book, I will venture to call a Token. ... In order that a Type may be used, it has to be embodied in a Token which shall be a sign of the Type, and thereby of the object the Type signifies. I propose to call such a Token of a Type an Instance of the Type. Thus there may be twenty Instances of the Type 'the' on a page.

I do not know whether Peirce ever supplemented his type-token distinction with the concept of "occurrence" as needed to make the point that, although the type 'the' has only one occurrence of the type 'e', the type 'e' occurs twice

\footnotetext{
${ }^{10}$ As important as this passage has become in modern linguistics, analytic philosophy, and logic I have been unable to find a single quotation of it besides the one by Ogden-Richards [33] copied here.
} 
in the type 'thee' and it is instantiated, betokened, or embodied ${ }^{11}$ (to use Peirce's term) twice in every token of the type tee-aitch-ee-ee. In order for two string occurrences in the same or different string-types to be occurrences of one and the same string-type it is necessary and sufficient for them to be "character-by-character identical", i.e., for them to have the same length, say L, and for the $n$th character occurrence in one to be an occurrence of the same character-type that the $n$th character occurrence in the other is an occurrence of, for $n$ between 1 and $L$.

Some authors explicitly make the three-part type-token-occurrence distinction without introducing special terminology for the occurrence relation. Lyons [29, Vol. 1, pp. 13-18] has a section called Type and token which discusses the three-way distinction while using the same expressions for token and occurrence. However, the present terminology is familiar to logicians, as pointed out in my "Meanings of word: type-occurrence-token [11, p. 117]". At some point in the history of logic the type-token dichotomy gave way to the type-token-occurrence trichotomy. Given Peirce's penchant for trichotomies and his logical creativity, one is led to speculate, even hope, that it was Peirce who made this discovery.

Template-text and instance. A scheme-template is a string type having indefinitely many tokens in Peirce's sense (Corcoran et al. [12, fn. 5, p. 638]). But none of the tokens of a scheme-template are instances of the schema. This is an ontological necessity and not a mere matter of convention like the fact mentioned above that no instance of a sentence schema is a schematemplate. In fact, every instance of a schema is a string type having its own tokens. As mentioned above, the word 'instance' is being used here, and throughout the modern literature on schemata, as a relation noun for a relation certain string types bear to certain schemas, not in Peirce's sense. The word 'token' is a relation noun for a relation certain macroscopic physical objects bear to certain abstract objects. The ontological status of a schemetemplate is the same as that of an instance of a schema. Moreover neither a schema nor a scheme-template is a common noun denoting the instances and neither is a proper name of a set of instances.

Some philosophers emphasize the ontological economies possible by using schemas rather than second-order axioms (see below). But, as is often

\footnotetext{
${ }^{11}$ Notice that Peirce says that the token embodies the type and is an instance of the type, not that the token is in or is a member of the type. He says that the type is a word in one sense of 'word'. There is no suggestion however slight that the type is to be identified with the huge and evolving class of its tokens. Of course, Peirce's theory-laden terminology can not be regarded as in any way binding on later logicians. Nevertheless, it is a gross but common mistake, perhaps an anachronism, to write as if Peirce and those who have adopted his terminology take the type to be the class of its tokens. This mistake occurs in otherwise respected and authoritative sources such as the 1993 New Shorter Oxford English Dictionary [32, p. 3441 of Volume 2] and the 1999 Cambridge Dictionary of Philosophy [1, pp. 936-937].
} 
the case, economies have their own costs. Rarely if ever do these philosophers present a full and objective discussion of the spectrum of "ontological commitments" entailed by the use of schemas. See Quine [39]. For example, number theory per se presupposes the existence of numbers, numerical functions and numerical properties, but it does not presuppose the existence of mathematical notation and it a fortiori does not presuppose the existence of the vast, intricate notational system that we call the language of number theory. Sometimes the use of schemas may decrease the ontological commitments of the object language while increasing those of the metalanguage, or at least not achieve any net savings.

Alternatives to schemas. Normally, the purpose for which a given logician uses a given schema goes far beyond simply specifying its instances. For example, Tarski's schema T has heuristic, pedagogical, rhetorical, and aesthetic effectiveness that is not overlooked by logicians that use it. Moreover, were specification of instances the sole purpose, it would be easy to do that without using a schema. For example, an instance of Tarski's schema T may be specified as the expression obtained from a given English sentence by prefixing to it the result of prefixing one of its English names to the eight-word string 'is a true sentence if and only if'. For example prefixing 'igh-spaceay-em is a true sentence if and only if' to 'I am'. Here the operation called prefixing adds a space - prefixing ay to bee yields ay-space-bee, that is ' $a$ b' not ay-bee, or 'ab'. In other contexts, it is convenient to use the same word for the operation of concatenating the first of two given strings with the second to yield a third string whose length measured in character-occurrences is the sum of the lengths of the given strings.

Truly subtle points. No discussion of the ontological status of schemata would be complete unless it at least recognized the thorny ontic issues concerning languages, both ideographic and phonographic, character-types, string-types, and the operation Tarski calls concatenation. Moreover, any discussion of schemata presupposes that some strings denote or name themselves or other strings, which begs the question of the ontological status of denoting - the relation that a string bears to that which it denotes. The issue here referred to as the ontological status of character-types and string-types is similar to the issues going back to Plato discussed as "the problem of universals". Tarski forthrightly addressed the issue in his 1933 truth-definition paper where he says that it raises "truly subtle points" [46, 48, pp. 156, 174]. Tarski's treatment, mentioned in the 1974 article "String Theory" (Corcoran et al. [12, footnote 5]), attempts to reduce a string-type to concrete material objects by "identifying" the abstract string-type with the class of its tokens. This class is taken to be an equivalence class induced by what Tarski calls the equiform relation which is defined as holding between tokens of the same 
shape $^{12}$. Tarski is well aware that this could undermine the plausibility of his theory of truth by calling into question some of the axioms of his metatheory - axioms that are entirely plausible when interpreted as concerning abstractions but which imply the existence of arbitrarily large material objects when taken as he suggests. For example, see Tarski's disclaimers [46, p. 31, fn. 3, p. 156, fn. 1, p. 174, fn. 2].

\$4. Schemas in the history of logic. The Greek word 'schema' was used in Plato's Academy for "[geometric] figure" and in Aristotle's Lyceum for "[syllogistic] figure". As mentioned above, the syllogistic figures fall under an interesting limiting case of "schema" in the senses of this article, as will be explained below. Each of the four traditional syllogistic figures is an argument schema. Kant used the words 'schema' and 'schemata' in a way only remotely related to the senses of this article.

Connotations. The articles schema through scheme in the 1971 Oxford English Dictionary [34, Compact Edition, Volume II, pp. 204-206] and the 1993 New Shorter Oxford English Dictionary [32, Volume 2, p. 2711] are certainly interesting to anyone concerned with the conceptual frameworks of modern linguistics, logic and philosophy - despite the fact that neither work recognizes the senses of the words 'schema', 'schemata', 'schematic', and 'scheme' most relevant to this article. ${ }^{13}$ What emerges from reading these articles is a vague connotation that must have been operative in the thinking of the people who coined the current range of meanings and in the thinking of those of us who found it easy, even natural, to accept the coinages. I seem to find the vague connotation of 'schema' and its cognates to be closer to "incomplete" than to "complete", closer to "potential" than "actual", closer to "apparent" than "real", closer to "abstract" than "concrete", and closer to "form" than to "matter". But I would not be surprised if others differ with me on such a subjective issue.

Other uses in logic. The above points are relevant to the concerns of this paper for several reasons not the least of which is that modern logicians, even those who are meticulous about using 'schema' in a technical sense, continue to use the cognates as standard non-technical English. For example, Tarski [43, p. 159] uses 'schema' in the context 'schema of the proof' in the sense of "style" or "form", Carnap [5, p. 1] says that an uninterpreted language "is not a language but a schema or skeleton of a language" and Goldfarb [21, p. 352] refers to ordinary non-logical constants as "schematic

\footnotetext{
${ }^{12}$ Even this requires modification in view of the fact that in some alphabets tokens of different character types have the same shape and differ in some other way. For example in some mathematical languages union, intersection, inclusion and subset are expressed by four characters whose tokens are all U-shaped.

${ }^{13}$ There are other uses of 'schema' and its cognates not recognized by the OED. For example, 'schema' has been used in the sense of a draft document to be discussed and revised (Encyclopedia Britannica [15, Vol. 6, p. 591]).
} 
placeholders" to emphasize the fact that they admit of reinterpretation. In the late 1890s, Hilbert said that a theory is a scaffolding or schema whose basic elements can be thought of anyway one likes.

Moreover, in the theory of recursive functions there are technical uses of 'schema' and its cognates that may seem temptingly close to the uses dealt with in this paper but are actually quite remote. Some of the clearest examples are found in the early papers by Kleene [25, pp. 239ff], [26, pp. 256ff]. Schemas are also used in computer science and engineering. According to Dr. E. Ray Lucas, formerly of IBM Research (per. comm.):

The development of linguistics and computer science has resulted in a proliferation in the use of schemas. Productions in formal grammars are schemas, a well-known example being the use of Backus-Naur Form (BNF) productions to specify programming languages. Another important use of schemas (called 'schemes') is in the specification of the structure of databases.

As late as 1969, the North-Holland Publishing Company's Dictionary of Symbols of Mathematical Logic [16] not only failed to devote a special paragraph to this concept but it did not even see fit to list the word 'schema' or any of its cognates in the index of subjects. Its index does list 'axiom schema' as occurring in one paragraph ( 72.1 on page 126$)$ where it occurs only once without comment or explanation.

The 1933 truth-definition paper (Tarski [42, pp. 157, 160, 172]), one of the first publications to prominently use a schema or to use the word 'scheme' in a sense close to this sense, has become the locus classicus for the subject [42, pp. 155, 156]. The word 'schema', or its plural 'schemata', is also, but less conspicuously, used by Tarski in the Pre-war period (Tarski [46], 63-64, $114,310,386,423)$. One of the earliest uses of the word 'schema' in logic is instructive and possibly of historical importance even though it takes the word in a different, but related sense. Russell in his influential 1919 Introduction to Mathematical Philosophy [41] writes on page 157: "A propositional function ... may be taken to be a mere schema, a mere shell, an empty receptacle for meaning, not something already significant." As in Russell's use of 'schema', it is clear that Tarski is not using 'scheme' as an established technical term nor is he presenting a stipulative definition. In both cases the authors are simply using the commonly available words to express their thoughts. Boole [3, pp. 33, 42] had already used 'logical scheme' and 'scheme of logic' in his discussion of ways of presenting Aristotle's syllogisms. And

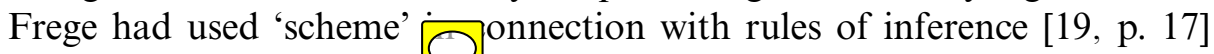
as did Gödel [20, p. 584] 2 ogicians know how awkward it would be to give a first-order formalized first-order logic without schemas. Surprisingly, we find no axiom schemas in Gödel's 1930 completeness paper: he uses second-order sentences with suppressed second-order quantifiers - strings that would at first glance appear to be schemas but which lack blanks or 
place-holders. It is clear that in none of these cases is a technical term being introduced for what we have been calling schemas. On the other hand it is just as clear that Church 1956 [6] takes 'schema' to be a technical term of logic. It would be interesting to determine when and how the word acquired its standing as an element of the technical vocabulary of logic. There are other issues to be settled: for example, whether cognates of 'schema' are the only words used in these senses as technical terms today, why it is that these words became accepted so widely and so quickly, and whether these senses were ever expressed using any other words.

In the past twenty-five years, the prominence and importance of schemas has become increasingly evident. For example, in Quine's Philosophy of Logic [39] schemas have a prominence not equaled in many previous books. For many years, Quine was especially active in exploring various schemas and their uses in logic and philosophy of logic: "On the Logic of Quantification" (Quine [37]) was especially influential. The most recent extended development and articulation of a Quinean approach may be found in Goldfarb's 2003 Deductive Logic [21].

Aristotle should probably be credited with introduction of argument schemas, or more explicitly, premise-conclusion argument-text schemas, although this point has not been settled in the minds of historians of logic. For example the two-premise argument text

\section{Polygon belongs-to-every multilateral.}

Multilateral belongs-to-every square.

Polygon belongs-to-every square.

is an instance of the argument-text schema

$$
\begin{gathered}
\mathrm{P} \text { belongs-to-every } \mathrm{M} \\
\mathrm{M} \text { belongs-to-every } \mathrm{S} \text {. } \\
\hline \mathrm{P} \text { belongs-to-every } \mathrm{S}
\end{gathered}
$$

called BARBARA, whose side condition includes requirements that (1) both occurrences of pee are to be filled with occurrences of one and the same common noun, (2) both occurrences of em are to be filled with occurrences of one and the same common noun other than the one used for pee, (3) both occurrences of ess are to be filled with occurrences of one and the same common noun other than the ones used for pee and em, and that (4) the expression 'belongs-to-every' is taken to express universal affirmative predication as in Prior analytics. There is no dispute that Aristotle used argument-text schemas similar to the one called BARBARA above. But it is disputable whether he was aware of the ontological presuppositions involved, e.g., that we are warranted in speaking of alphabetic characters as existent individuals, that the common nouns of a language form a welldefined totality, or that a schema may seem to be a linguistic unity that 
ontologically transcends the plurality of its instances in much the same way that a Platonic form transcends the plurality of individuals that participate in it.

Every argument text that is an instance of BARBARA is in the first syllogistic figure, i.e., is an instance of the argument-text schema

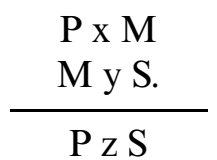

whose side condition includes requirements that (1) both occurrences of pee are to be filled with occurrences of one and the same common noun, (2) both occurrences of em are to be filled with occurrences of one and the same common noun other than the one used for pee, (3) both occurrences of ess are to be filled with occurrences of one and the same common noun other than the ones used for pee and em, and that (4) the occurrences of ecks, why, and zee are to be filled with occurrences of 'belongs-to-every', 'belongs to no', 'belongs to some' or 'does not belong to every' taken to express the categorical predications as in Prior analytics. The other three figures also correspond to schemas. Notice that the four figure schema-templates are composed entirely of blanks and thus fall under a limiting case of schemas. ${ }^{14}$

Fraenkel [18] may have been more important than is usually thought as far as the introduction of the use of axiom schemas in modern theories for delimiting an infinity of axioms. But the full history has yet to be written. Quine [38, p. 89] writes that "the practice of describing an infinity of axioms dates only from von Neumann [31]". One year later Kleene [27, p. 140] also credits von Neumann with the method of using axiom schemata in the formalization of logic. A few years later Church [6, p. 158] also makes the assertion that this use of axiom schemas was introduced by von Neumann in 1927.

\footnotetext{
${ }^{14}$ The original 1999 Corcoran article [9] on schemata does not discuss the history of the subject and consequently omits this theoretically and historically interesting if arcane point that I was unaware of at the time. The first couple of published versions of the 2004 Corcoran article [10] on schemata do indeed discuss the history of the subject, but nevertheless they also omit this point. Even worse, although they cited the Aristotelian moods (tropoi) as schemas, they explicitly denied that the four traditional figures (schemata) were schemas in the modern sense. At the time of the publication of the very first of these, it did not occur to me that there could be schema-templates composed entirely of blanks and containing no characters from the underlying object language. But as soon as I saw it in print, I realized my mistake. Instead of immediately making the correction, I decided to wait until more mistakes were brought to my attention so that the corrections could be done all at once. In addition, I was curious whether someone else would notice the mistake. Indeed, at least one person did - my long-time friend and former colleague Mary Mulhern (per. comm.). Even if the line is regarded as a meaningful character in the argument schema, the three-character sentence schemas occurring in it are schemas composed entirely of blanks. This was first pointed out by Joaquin Miller (per. comm.).
} 
It is hard to date self-conscious use of the word 'schema' in the modern sense. As mentioned above, Russell's 1919 Introduction to Mathematical Philosophy [41] uses it casually on page 157 to describe "propositional functions" or, roughly, interpreted open sentences in Quine's sense.

Since first-order number theory cannot be finitely axiomatized, the mathematical induction schema is used to effectively specify an infinite number of axioms: 'If zero is such that ... and the successor of every number such that $\ldots$ is also such that ..., then every number is such that ...', where the four blanks are to be filled in with one and the same arithmetic open sentence such as 'it is zero or it is a successor', 'it precedes its own successor' or 'it is finite'. Every instance of the mathematical induction schema is implied by the second-order principle of mathematical induction: every property that belongs to zero and that also belongs to the successor to every number to which it belongs, also without exception belongs to every number. In other words, the principle of mathematical induction is that in order for a given property to belong to every number it is sufficient for it to belong to zero and for it to belong to the successor of every number to which it belongs (Russell [41, p. 5, 6]).

Contrary to what would at first be thought, the principle of mathematical induction is a consequence of the set of all instances of the mathematical induction schema, but of course, it is not a consequence of the set of all firstorder instances. In fact, the principle of mathematical induction is logically equivalent to a certain single instance of the schema, where the open sentence is taken to be: 'is such that it has every property that belongs to zero and to the successor to every number to which it belongs'. To be sure, the set of first-order instances is weaker than the second-order principle, i.e., it does not logically imply the second-order principle, as is shown in any advanced logic text, e.g., Boolos et al. [4].

Sometimes the blanks in a schema are marked by variables; thus, in the minds of some, blurring the crucial distinction between, on one hand, an open sentence, such as ' $(\mathrm{x}+\mathrm{y})=(\mathrm{y}+\mathrm{x})$ ' whose object-language numerical variables ecks and wye range over the numbers and, on the other a schema such as the number-theoretic commutativity schema ' $(\mathrm{X}+\mathrm{Y})=(\mathrm{Y}+\mathrm{X})$ ' whose template-text involves the side condition that the two occurrences of ecks are to be replaced by two occurrences of one and the same numeral and likewise for the two occurrences of wye. One critical distinction is that an open sentence is a formula of the object language whose variables range over the universe of discourse and which is satisfied (or not) by sequences of objects in that universe, whereas the variables occurring in the schema's template-text are what Church calls syntactic variables [6, Sects. 08, 27, 30 and 33], variables that are not in the object language vocabulary but rather belong to the metalanguage and which range over syntactic objects, strings of characters, not entities such as numbers in the universe of discourse of 
the interpretation of the object-language. Church [6, Sect. 27] makes further related points. In the case of the variables occurring in schema-templates it is not clear whether there is any need or justification in saying that they "range over" syntactic objects. What is clear and sufficient is that they have syntactic objects as substituents. They are not objectual variables, but it might be justifiable to call them substitutional variables. It does not help that the expression 'dummy letter' is sometimes applied to variables and sometimes to place-holders.

As Church has emphasized (e.g., [6, p. 59]), rigorous metamathematical treatment of schemas requires use of formalized or logically perfect languages and it requires an axiomatized theory of strings as found for the first time in the 1933 Tarski truth-definition paper [48, pp. 152-256]. For more on the history, philosophy, and mathematics of this important but somewhat neglected field, string theory or pure syntax, see the article "String theory" (Corcoran et al. [12]).

§5. Argument-texts and argument-text schemas. An argument, or more properly, an argument-text is a two part system composed of a set of sentences called the premises and a single sentence called the conclusion. Of the various ways of presenting an argument perhaps the one least open to misinterpretation is the premises-line-conclusion format which consists in listing the premises followed by a line followed by the conclusion. ${ }^{15}$ Below are four examples.

A3 Every circle is a polygon.

Every triangle is a circle.

Every square is a triangle.

Every square is a polygon.

A2 Every circle that is a square that is a polygon is a triangle. Every circle that is a square that is not a polygon is a triangle.

Every square that is a circle is a triangle.

\footnotetext{
${ }^{15} \mathrm{~A}$ presentation of an argument-text is nothing more than a way of describing the argument-text. It does not include an assertion that the argument-text expresses a valid argument. Therefore, any sign indicating validity, or (what amounts in this context to the same thing) an implication, is inappropriate and misleading. Moreover, it does not include an assertion of the truth of any propositions. Consequently, it is even more inappropriate and misleading to attach the word 'therefore' or any of its synonyms, abbreviations, or symbolizations. These points have been made in various places including my 1972 article "Conceptual Structure of Classical Logic"[8]. In view of the fact that 'therefore', like 'since' and 'because', is a sentential connective, its use in a presentation of an argument-text invites a confusion of "argument" with "sentence" - not to mention that it is both modal and non-truth-functional.
} 
A1 No square is a triangle.

No triangle that is a circle is a square.

A0

No triangle that is a square is not a triangle.

There is no limit to the number of premises in an argument-text. In fact, zero-premise argument-texts like A0 above have come to play a surprisingly important role in modern logic. Likewise important are infinite-premise argument-texts, especially the so-called omega argument-texts illustrated below.

A Zero is not the successor of zero.

Zero is not the successor of the successor of zero.

Zero is not the successor of the successor of the successor of zero.

Zero is not the successor of any number.

As usual, an argument is said to be valid if the conclusion is a logical consequence of, or follows logically from, its premise-set. When it is clear how the sentences are to be interpreted, the same terminology can be applied to argument-texts, although strictly speaking it is only an argument expressed by an argument-text under a given interpretation that can properly be said to be valid or invalid, not the argument-text itself. This parallels the familiar point that when it is clear how the sentences being discussed are to be interpreted the sentences themselves can be said to be true or false, although strictly speaking it not the sentence per se but the proposition it expresses under an interpretation that is true or false.

An argument schema is said to be pan-valid if all of its instances are valid, pan-invalid if all are invalid, and neutro-valid if some but not all of its instances are valid, i.e., if it is neither pan-valid nor pan-invalid. See e.g., Cohen-Nagel [7, pp. xxxiv-xxxvii]. The advantages of using the same words to discuss arguments and argument-texts are evident as are the disadvantages. For example, it might lead to confusing argument-schemas with what the 1993 edition of Cohen-Nagel [7, pp. xxxi-xxxvii] calls argument-patterns and argument-forms. Also see [1, pp. 511-512].

Just as the schema-template of a sentence-schema may be thought of as obtained from a sentence by replacing various constituents with placeholders of one sort or another, the schema-template of argument-textschema may be thought of as obtained from an argument-text by replacing various constituents with place-holders. Rules of inference are associated with argument-text schemas and axiom schemas can be thought of as zeropremise argument-text schemas. 
Acknowledgements. This article originated in the early 1990s with a series of meetings of the Buffalo Logic Colloquium dedicated to the Buffalo Logic Dictionary Project. It owes much to those in attendance, especially to G. Boger, R. Dipert, P. Hare, K. Herreid, L. Jacuzzo, J. Kearns, W. Lawvere, J. McNabb, J. Sagüillo, B. Smith and R. Vesley. In very near its present form it was discussed twice by the Buffalo Logic Colloquium, once in 2004 and again in 2005 after corrections and other minor changes.

The first publication arising from the project was the article "Scheme" in the 1999 second edition of The Cambridge Dictionary of Philosophy [1], edited by Robert Audi. Readers of that short piece and this do not need to be told that I have learned a lot about the subject since then. Therefore, I deem it near-miraculous that no mistakes in the 1999 article have yet come to light, unless it was a mistake that I did not explicitly distinguish the templatetext (a string containing at least one blank) from the "template", a kind of abstract form or mold. The ambiguous word 'template' whose meanings are vague is used in the 1999 article for a genus of which the schemes form a species.

The template-text-versus-template distinction, only implicit in that article, was made explicit by an anonymous referee-editor of the second publication arising from this project, namely the 2004 article "Schema" in the Stanford Encyclopedia of Philosophy [10]. Of the several scholars who suggested improvements in previous drafts and versions, I want to mention for special thanks those already mentioned above, especially J. Sagüillo, and also R. Grandy, D. Hitchcock, A. Kanamori, F. Nabrasa, A. Preus, J. Miller, M. Mulhern, R. Santos, M. Scanlan and an anonymous referee for The Bulletin of Symbolic Logic. Above all I thank my friend of more than forty years Dr. E. Ray Lucas, who informed me of the role of schemata in computer science and reminded me of several relevant points that we had learned together in the early 1960's in Baltimore, Madison and Berkeley, but that I had almost forgotten.

\section{REFERENCES}

[1] R. Audi (editor), The Cambridge Dictionary of Philosophy, 2nd ed., Cambridge University Press, Cambridge, 1995/1999.

[2] P. Bernays, Axiomatic Set Theory, Dover, New York, 1958/1991.

[3] G. Boole, The Mathematical Analysis of Logic, Macmillan, Cambridge, 1847.

[4] J. Boolos, G. Burgess and R. JefFrey, Computability and Logic, Cambridge University Press, Cambridge, 2002.

[5] R. CARnAP, Introduction to Symbolic Logic and its Applications, Dover, New York, $1954 / 1958$.

[6] A. Church, Introduction to Mathematical Logic, Princeton University Press, Princeton, 1956.

[7] M. CoHEn and E. NAGEL, Introduction to Logic, 2nd ed., Hackett, Indianapolis, 1993, 1st ed. Harcourt, Brace and World, New York, 1962, originally published as Book I of $\boldsymbol{A n}$ 
Introduction to Logic and Scientific Method, Harcourt, Brace, and Company, New York, 1934.

[8] J. Corcoran, Conceptual structure of classical logic, Philosophy and Phenomenological Research, vol. 33 (1972), pp. 25-47.

[9] — Scheme, In Audi [1], 1999.

[10] - Schema, Stanford Encyclopedia of Philosophy, 2004.

[11] - Meanings of word: type-occurrence-token, this Bulletin, vol. 11 (2005), p. 117.

[12] J. Corcoran, W. Frank, and M. Maloney, String theory, The Journal of Symbolic Logic, vol. 39 (1974), pp. 625-637.

[13] M. DAvis, Computability and Unsolvability, 2nd ed., McGraw-Hill, Dover, New York, $1958 / 1980$.

[14] , The Undecidable, Raven Press, Hewlett, 1965.

[15] Encyclopedia Britannica, Chicago, London, Toronto, 1953.

[16] R. Feys and F. Fitch (editors), Dictionary of Symbols of Mathematical Logic, NorthHolland Publishing Company, Amsterdam, 1969.

[17] A. FraenKel, Introduction, [2], 1958/1991.

[18] - The notion "definite" and the independence of the axiom of choice, In Heijenoort [22], 1967, pp. 284-289.

[19] G FREge, Philosophical and Mathematical Correspondence, Chicago University Press, Chicagd 10 .

[20] K. GöDEL, The completeness of the axioms of the functional calculus of logic, In Heijenoort [22], 1967, pp. 582-591.

[21] W. Goldfarb, Deductive Logic, Hackett, Indianapolis, 2003.

[22] J. van Heijenoort (editor), From Frege to Gödel, Harvard University Press, Cambridge, MA, 1967.

[23] J. Herbrand, Logical Writings, (W. Goldfarb, Tr. Goldfarb, and van J. Heijenoort, ecitors), Harvard University Press, Cambridge, MA, 1971.

[24] R. Hughes, Philosophical Companion to First-order Logic, Hackett, Indianapolis, 1993.

[25] S. KLEENE, General recursive functions of natural numbers, Mathematische Annalen, vol. $112(1936 / 1965)$, no. 5, pp. 727-742, also, in [14], pp. 237-254.

[26] - Recursive predicates and quantifiers, Transactions of the American Mathematical Society, vol. 53 (1943/1965), pp. 41-73, reprinted in [14], pp. 255-287.

[27] — Introduction to Metamathematics, Van Nostrand, Princeton, 1952.

[28] L. Linsky, Semantics and the Philosophy of Language, University of Illinois Press, Urbana, 1952.

[29] J. Lyons, Semantics, Cambridge University Press, Cambridge, 1977, 2 Vols.

[30] Merriam-Webster's Collegiate Dictionary, Merriam-Webster, Springfield, MA, 2000 .

[31] J. von Neumann, Zur Hilbertschen Beweistheorie, Mathematische Zeitschrift, vol. 26 (1927), pp. 1-46.

[32] New ShOrter Oxford English Dictionary, Oxford University Press, Oxford, 1993.

[33] C. K. Ogden and I. A. Richards, The Meaning of Meaning, 1923, reprint of the eighth edition, Harcourt, New York.

[34] Oxford English Dictionary, compact ed., Oxford University Press, Oxford, 1971.

[35] C. S. PeIrCe, Prolegomena to an apology for pragmaticism, Monist, vol. 16 (1906), pp. $492-546$.

[36] - Collected papers of Charles Sanders Peirce, (C. Hartshorne and P. Weiss, editors), Harvard University Press, Cambridge, 1933. 
[37] W. QuINE, On the logic of quantification, The Journal of Symbolic Logic, vol. 10 (1945), pp. $1-12$.

[38] — Mathematical Logic, Harvard University Press, Cambridge, MA, 1951.

[39] — Philosophy of Logic, Harvard University Press, Cambridge, MA, 1970/1986.

[40] P. Rosenbloom, Elements of Mathematical Logic, Dover, New York, 1950.

[41] B. Russell, Introduction to Mathematical Philosophy, George Allen and Unwin, London, 1919.

[42] A. TARSKI, The concept of truth in the languages of the deductive sciences, Prace Towarzystwa Naukowego Warszawskiego, Wydzial III Nauk Matematyczno-Fizycznych, vol. 34 (1933), reprinted in [50], pp. 13-172; expanded English translation in [48], pp. 152-278.

[43] - Introduction to logic and to the methodology of deductive sciences, Dover, New York, 1941/1946/1995, Trans. O. Helmer.

[44] - Introduction to logic and to the methodology of deductive sciences, Oxford University Press, New York, 1941/1994, ed. with preface and biographical sketch of the author by J. Tarski.

[45] - The semantic conception of truth, Philosophy and Phenomenological Research, vol. 4 (1944), pp. 341-376, in [28], pp. 13-49.

[46] - Logic, Semantics, Metamathematics, papers from 1923 to 1938, Oxford University Press, Oxford, 1956.

[47] —, Truth and proof, Scientific American, (1969), also, in [24], pp. 101-125.

[48] — Logic, Semantics, Metamathematics, papers from 1923 to 1938, 2nd ed., Hackett, Indianapolis, 1983, edited with introduction and analytic index by J. Corcoran.

[49] R. Vaught, Axiomatizability by a schema, The Journal of Symbolic Logic, vol. 32 (1967), pp. 473-479.

[50] J. Zygmunt (editor), Alfred Tarski, Pisma Logiczno-Filozoficzne, 1 Prawda, Wydawnictwo Naukowe PWN, Warsaw, 1995.

\author{
UNIVERSITY OF BUFFALO \\ DEPARTMENT OF PHILOSOPHY \\ BUFFALO, NY 14260-4150, USA \\ E-mail: corcoran@buffalo.edu
}

